\title{
General Form of Model-Free Control Law and Convergence Analyzing
}

\author{
Xiuying Li, ${ }^{1,2}$ Guanghui Wang, ${ }^{1}$ and Zhigang Han ${ }^{1,2}$ \\ ${ }^{1}$ Key Laboratory of Electronic Engineering, College of Heilongjiang Province Heilongjiang University, Harbin 150080, China \\ ${ }^{2}$ Department of Automation, School of Electronic Engineering, Heilongjiang University, Harbin 150080, China
}

Correspondence should be addressed to Zhigang Han, zgh3468@yahoo.com.cn

Received 31 July 2011; Revised 25 March 2012; Accepted 12 April 2012

Academic Editor: Chengyu Cao

Copyright ( $) 2012$ Xiuying Li et al. This is an open access article distributed under the Creative Commons Attribution License, which permits unrestricted use, distribution, and reproduction in any medium, provided the original work is properly cited.

\begin{abstract}
The general form of model-free control law is introduced, and its convergence is analyzed. Firstly, the necessity to improve the basic form of model free control law is explained, and the functional combination method as the approach of improvement is presented. Then, a series of sufficient conditions of convergence are given. The analysis denotes that these conditions can be satisfied easily in the engineering practice.
\end{abstract}

\section{Introduction}

The model-free control method we proposed is of great significance in theory and practical applications. The overall framework to design the model-free control law is given in $[1,2]$, where the following characteristics are also described. Firstly, it is an adaptive control law not only in parameter but also in structure. Secondly, it is a nonlinear controller by breaking through the linear framework constraints of PID [3]. Thirdly, it is designed by the new thought of modeling and control, that is, "the integration of modeling and control" approach [4]. Since the model-free control method has been put forward, it has been applied to the oil refining $[5,6]$, chemical $[7,8]$, power [9], glass $[10]$, and other industries. References [11,12] give a brief overview of its application and progress. In the actual context of the industrial process, the unit control issue of complex systems is analyzed in [13], the cascade form and its application effects are analyzed in [14], and the control functions of model-free control law are analyzed in [15-17]. It has been illustrated that the model-free control law has a strong ability of antidisturbance [18] and the ability to overcome the large time-delay [19], as well as a strong decoupling performance [20]. In general, the practical application results are richer than theory research of model-free control law. It is imperative to do more in-depth theoretical study for further application of this method.

We published the article [4] with the topic of "the integration approach of modeling and adaptive control" in 2004, in which we put forward the "universal model" and "the basic form of model-free control law" under the idea of the integration of modeling and control and analyzed its convergence. The control results are satisfactory for simple objects, but not pleasing for complex. So in the engineering application, the model-free control law is used not in its basic form, but some improved forms. These improved forms of model-free control law have received more satisfactory results for the objects difficult to control. To lay the theoretical basis for practical applications, it is necessary to give the theoretical analysis for these improved forms. This paper is written under such circumstances.

For the universality of the theoretical research, all the improved model-free control law can be written in a unified form, which called as the general form of model-free control law.

In this paper, the necessity and method of the general form of model-free control law are discussed firstly, then the convergence of the control law is analyzed, and some convergent conditions are also given. It can be seen as continued and expanded of the reference [4]. 


\section{Necessity and Approach of Improving Model-Free Control Law}

In the literature [4], we had discussed the integration approach of modeling and adaptive control and obtained the integration framework of modeling and feedback control for the systems without model, which are given as follows.

(1) Based on observation data and the universal model of system S

$$
y(k)-y(k-1)=\varphi(k-1)^{T}\{u(k-1)-u(k-2)\},
$$

we can get the estimation value $\hat{\varphi}(k-1)$ of $\varphi(k-1)$ by using some appropriate estimation method.

(2) To look for the one step prediction value $\hat{\varphi}^{*}(k)$ of $\hat{\varphi}(k-1)$, a simple way is to take

$$
\hat{\varphi}^{*}(k)=\hat{\varphi}(k-1) .
$$

We still write $\hat{\varphi}^{*}(k)$ as $\hat{\varphi}(k)$ for seeking control law, and based on universal model, we can derive the control law:

$$
u(k)=u(k-1)+\frac{\lambda_{k}}{a+\|\hat{\varphi}(k)\|^{2}} \hat{\varphi}(k)\left\{y_{0}-y(k)\right\} .
$$

(3) Under the effect of control law (3), we can obtain a new output $y(k+1)$ of system $\mathrm{S}$. And then we get a group of new data $\{y(k+1), u(k)\}$.

On the basis of this new group data, we repeat the procedure (1), (2), and (3), can also get the new data $\{y(k+$ $2), u(k+1)\}$, and continue to do it. It can be proven that, as long as the system $S$ satisfies certain conditions, the output $y(k)$ of system $\mathrm{S}$ will be gradually approaching to $y_{0}$ under such procedures.

We call the control law (3) as the basic form of model-free control law. In (3), $\lambda_{k}$ is named as the control parameter, $a$ is a small positive number, which can avoid the denominator being zero, $\hat{\varphi}(k)$ is the estimation value of the characteristic parameter $\varphi(k)$.

It is not difficult to find in practical application, the control results for the simple objects of control law (3) are satisfied, but the results for more complex objects cannot be satisfactory. This also can be seen by the following examples.

Consider the system described by the following model:

$$
y(k)=1.3 y(k-1)-0.42 y(k-2)+0.5 u(k-1) .
$$

It is controlled by using the basic form of model-free control law (3). Let $\lambda_{k}=1, \hat{\varphi}(k)=100, A=1$, and the setting value is $y_{0}=70$. The control result is shown in Figure 1.

When the system is changed into

$$
y(k)=1.3 y(k-1)-0.31 y(k-2)+0.5 u(k-1),
$$

we still use the aforementioned control law to control this system (parameters remain the same). The result is shown in Figure 2.

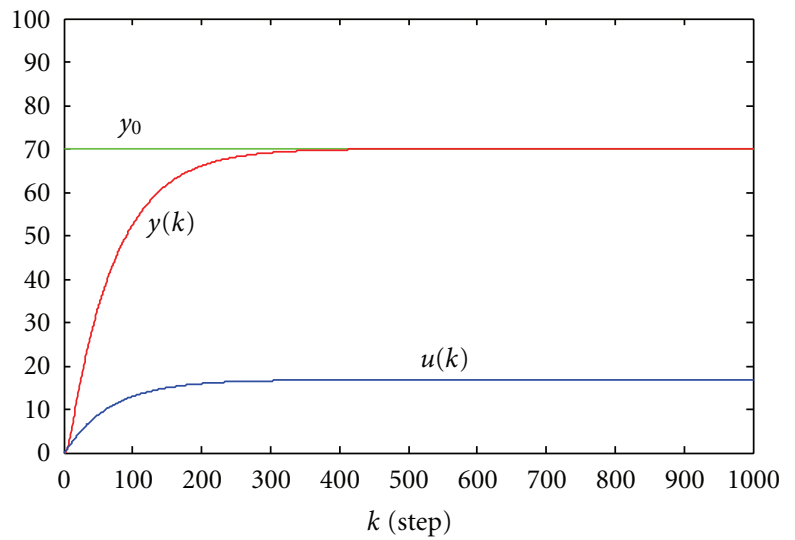

FIGURE 1: Control result of system (4).

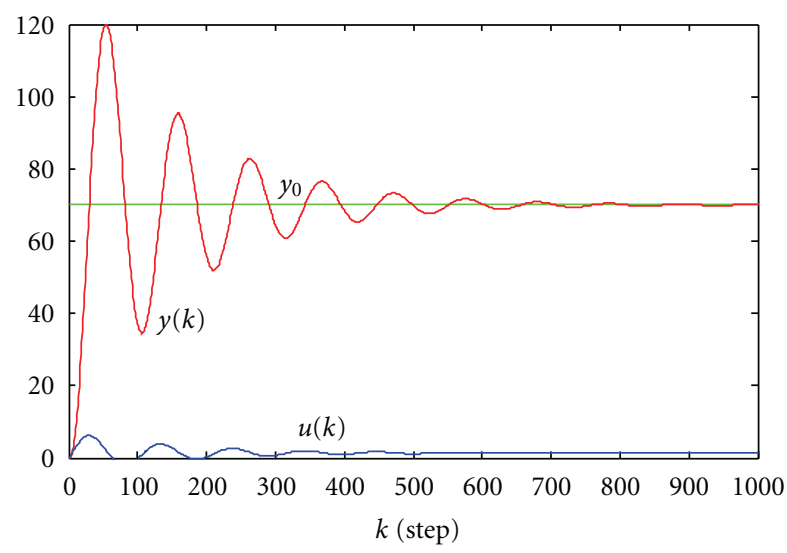

Figure 2: Control result of system (5).

This case shows that, when the performance of controlled system changes, the control result by using the control law (3) can not be satisfactory.

If the system is changed into

$$
y(k)=1.3 y(k-1)-0.28 y(k-2)+0.5 u(k-1),
$$

then the control result is divergence under the effect of control law (3) (graphics is omitted). This confirms the above judgment. Thus it is necessary to improve the control law (3).

The way of improvement is to add functional combination part $D\left[Y_{k-1}^{k-n}, U_{k-1}^{k-m}, \theta, k\right]$ on the basic form of model-free control law.

The functional combination part $D\left[Y_{k-1}^{k-n}, U_{k-1}^{k-m}, \theta, k\right]$ is obtained by the functional combination approach for controller's design $[1,2]$. The starting point is that the controller is derived from the controlled object's functional requests to the control law but does not depends on the mathematical model of the controlled object. The controlled system (object) has some basic requirements to the control law, typically such as: "overcoming deviation" and "convergence accelerating", which can be explained from Figure 2. The difference between the maximum value of system responds and the set value be smaller, the performance of the system is the better. We call this function of control law as "overcoming 
deviation". Moreover, the time of the system achieved the set value be shorter, the convergence of the system is the better. We call this function of control law as "convergence accelerating".

So the procedure of controller's design is to find the representations of these functions and combine them in some proper way. Practice shows that there will be great differences among the controllers' performances if these representations of the functions and the combination modes are different. So the basic contents of the functional combination method are (1) to find all kinds of functions that the controlled object requests, which are called as element function set; (2) to give each basic function (called element function) a kind of representation, best of all in the algorithmic form; (3) to combine these functions in some optimal way.

In the application scene, we call the procedures to find the proper functional combination as the configuration of model-free control law. For a different controlled object, the configuration process is also different; thus we can obtain the model-free control law applied to the controlled object.

The improved one is called as the general form of modelfree control law. There are two expressions of it.

(1) The form easy to analyze:

$$
\begin{aligned}
u(k)= & u(k-1)+\frac{\lambda_{k}}{a+\|\hat{\varphi}(k)\|^{2}} \hat{\varphi}(k) \\
& \times\left\{A+D\left(Y_{k-1}^{k-n}, U_{k-1}^{k-m}, \theta, k\right)\right\}\left(y_{0}-y(k)\right),
\end{aligned}
$$

where $D(\cdots)$ is a proper function, which denotes the functional combination part of control law, and:

$$
\begin{gathered}
Y_{k-1}^{k-n}=\left\{y_{0}, y(k-1), \ldots \ldots, y(k-n)\right\} \\
U_{k-1}^{k-m}=\{u(k-1), \ldots \ldots, u(k-m)\}
\end{gathered}
$$

$a$ is a small positive number, $A$ and $\theta$ are the configuration parameters, $A>0, \theta$ is a nonnegative vector, that is, all of its components are nonnegative. $m$ and $n$ are positive integers.

(2) The form easy to apply:

$$
\begin{gathered}
u(k)=u(k-1)+\frac{\lambda_{k}}{a+\|\hat{\varphi}(k)\|^{2}} \hat{\varphi}(k) \\
\left\{A\left(y_{0}-y(k)\right)+G\left(Y_{k-1}^{k-n}, U_{k-1}^{k-m}, \theta, k\right)\right\},
\end{gathered}
$$

where $G(\cdots)$ is a proper function, and it is the functional combination part of the control law.

Obviously the two forms can mutually convert. This paper will analyze the control law (7). Therefore, assume that the function $D\left[Y_{k-1}^{k-n}, U_{k-1}^{k-m}, \theta, k\right]$ satisfies the following conditions.

(1) When $\theta=0$, we have

$$
D\left[Y_{k-1}^{k-n}, U_{k-1}^{k-m}, 0, k\right]=0 .
$$

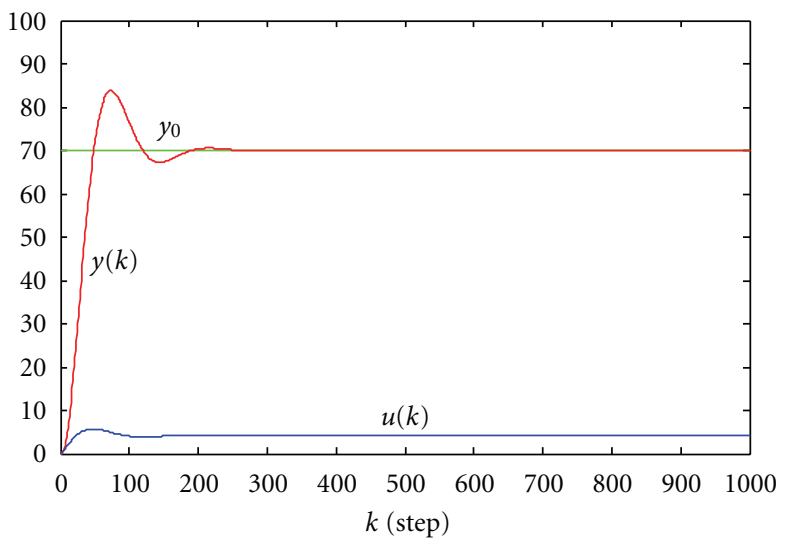

Figure 3: Control result of system (11).

(2) $D\left[Y_{k-1}^{k-n}, U_{k-1}^{k-m}, \theta, k\right]$ is a continuous function of $\theta$, and the continuity to other variable is consistent.

To control the system,

$$
y(k)=1.3 y(k-1)-0.31 y(k-2)+0.5 u(k-1)
$$

by using the general form of model-free control law, we still let $\lambda_{k}=1, \hat{\varphi}(k)=100$ and $A=1$ and select the appropriate parameter $\theta$. The control result is shown in Figure 3 .

It can be seen that the functional combination part plays a huge role in improving the control performance. This further confirms the necessity to improve the basic form of model-free control law.

Further, Let us give an application example. We have introduced a model-free control scheme with functional combinations for the propylene flow system in the carbonyl compose reactor of fourth-octanol device of the second chemical plant of the Daqing Petrochemical Company. The controlled system is a complex system with large time delay and strong disturbances. Under the control of DCS of the Honeywell Corporation, many sets of this system cannot be controlled steadily. We change the original DCS with model-free control method and have obtained good effects in practice.

The 24-hour scene record plan before and after the model-free control law applied is given in Figure 4, from which we can see that the model-free control method can obtain a good control performance.

We had analyzed the composition of functional combination part in [21]. In this paper, we will focus on the convergence after adding functional combination part to the basic form of model-free control law.

\section{Convergence Analysis of General Form of Model-Free Control Law}

We will need the following lemma in the discussion. 


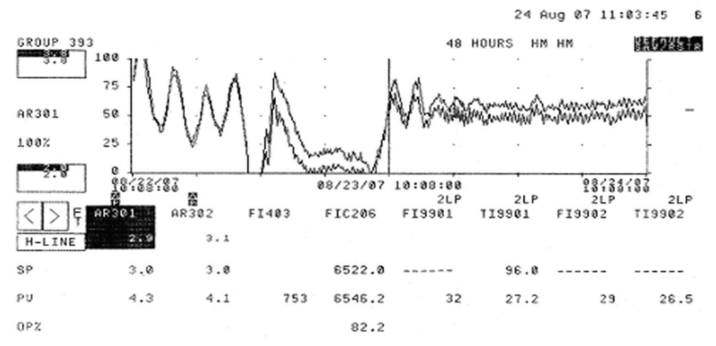

FIGURE 4: The scene record plan of model-free control method.

Lemma 1. For $B>0$, there exists the constant vector $\theta_{0}>0$, such that when the configuration parameter $\theta$ satisfies $0 \leq$ $\theta<\theta_{0}$, the functional combination part $D\left[Y_{k-1}^{k-n}, U_{k-1}^{k-m}, \theta, k\right]$ constantly holds:

$$
B>\left|D\left(Y_{k-1}^{k-n}, U_{k-1}^{k-m}, \theta, k\right)\right|
$$

In fact, from conditions (1) and (2) that the function $D\left[Y_{k-1}^{k-n}, U_{k-1}^{k-m}, \theta, k\right]$ satisfied, it can be concluded that is for $Y_{k-1}^{k-n}, U_{k-1}^{k-m}$, and $k$, we consistently have

$$
\lim _{\theta \rightarrow 0} D\left[Y_{k-1}^{k-n}, U_{k-1}^{k-m}, \theta, k\right]=0 .
$$

So for $B>0$, there must exist $\eta>0$, such that when $\|\theta\|<\eta$, we constantly have

$$
B>\left|D\left(Y_{k-1}^{k-n}, U_{k-1}^{k-m}, \theta, k\right)\right| .
$$

Then (12) holds as long as we choose $\theta_{0}$ to satisfy $\left\|\theta_{0}\right\|<$ $\eta$. We call the configuration parameter $\theta$ that satisfies the above lemma as being useable.

In order to design the model-free control law, we have discussed the so-called linearization problem of nonlinear dynamic systems in [22], where we illuminate that the following nonlinear model

$$
y(k)=f\left[Y_{k-1}^{k-n}, u(k-1), U_{k-2}^{k-m}, k\right]
$$

can be described in real time by the following universal model:

$$
y(k)-y(k-1)=\varphi(k-1)^{T}\{u(k-1)-u(k-2)\},
$$

combining with the control law in the sense of the inputoutput equivalence. Obviously we can rewrite the above formula as the following:

$$
y(k+1)-y(k)=\varphi(k)^{T}\{u(k)-u(k-1)\} .
$$

According to the universal model, we can prove the following convergence theorem.

Theorem 2. Consider the control law:

$$
\begin{aligned}
u(k)= & u(k-1)+\frac{\lambda_{k}}{a+\|\hat{\varphi}(k)\|^{2}} \hat{\varphi}(k) \\
& \times\left\{A+D\left(Y_{k-1}^{k-n}, U_{k-1}^{k-m}, \theta, k\right)\right\}\left(y_{0}-y(k)\right) .
\end{aligned}
$$

Suppose that there exists $N>0$, such that, when $k>N, \varphi(k)$ and its estimation value $\hat{\varphi}(k)$ constantly satisfy the relation:

$$
\begin{aligned}
& \varphi(k)-\hat{\varphi}(k)=\mathcal{E}(k) \\
& -\frac{\alpha}{2} \leq \mathcal{E}(k)^{\tau} \hat{\varphi}(k) \leq \frac{\alpha}{2}, \\
& \alpha \leq\|\hat{\varphi}(k)\|^{2} \leq \beta,
\end{aligned}
$$

where $\alpha$ and $\beta$ are both positive constants. Then there exists the constant $\lambda_{k}$ in the control law (18), such that for $A>0$, when the configuration parameter $\theta$ is useable, the output of the controlled system under the effect of this control law constantly holds:

$$
\lim _{h \rightarrow \infty} y(k+h)=y_{0}
$$

Proof. In the control law (18), let

$$
F(k)=A+D\left(Y_{k-1}^{k-n}, U_{k-1}^{k-m}, \theta, k\right) .
$$

Because the configuration parameter $\theta$ is useable, for the given $B>0(A>B)$, there exists the constant vector $\theta_{0}>0$ such that, when the configuration parameter $\theta$ satisfies $0 \leq$ $\theta<\theta_{0}$, we have

$$
B>\left|D\left(Y_{k-1}^{k-n}, U_{k-1}^{k-m}, \theta, k\right)\right| ;
$$

that is,

$$
B>D\left(Y_{k-1}^{k-n}, U_{k-1}^{k-m}, \theta, k\right)>-B
$$

Properly choose $A$ to satisfy $A-B=\rho>0$; then we have

$$
A+B>A+D\left(Y_{k-1}^{k-n}, U_{k-1}^{k-m}, \theta, k\right)>A-B=\rho .
$$

Let $F(k)=A+D\left(Y_{k-1}^{k-n}, U_{k-1}^{k-m}, \theta, k\right)$; thus

$$
A+B>F(k)>\rho>0 .
$$

Now the control law (18) is changed into

$$
u(k)=u(k-1)+\frac{\lambda_{k} F(k)}{a+\|\hat{\varphi}(k)\|^{2}} \hat{\varphi}(k)\left\{y_{0}-y(k)\right\} .
$$

Let

$$
\Delta_{k}=\frac{\varphi(k)^{T} \hat{\varphi}(k)}{a+\|\hat{\varphi}(k)\|^{2}} .
$$

Notice that

$$
\Delta_{k}=\frac{\varphi(k)^{T} \hat{\varphi}(k)}{a+\|\hat{\varphi}(k)\|^{2}}=\frac{\|\hat{\varphi}(k)\|^{2}+\varepsilon(k)^{T} \hat{\varphi}(k)}{a+\|\hat{\varphi}(k)\|^{2}} .
$$

Therefore

$$
0 \leq \frac{\|\hat{\varphi}(k)\|^{2}-\alpha / 2}{\|\hat{\varphi}(k)\|^{2}+a} \leq \Delta_{k} \leq \frac{\|\hat{\varphi}(k)\|^{2}+\alpha / 2}{\|\hat{\varphi}(k)\|^{2}+a} .
$$


We can derive from (17) and (25) that

$$
\begin{aligned}
y(k+1)-y(k) & =\varphi(k)^{T}[u(k)-u(k-1)] \\
& =\varphi(k)^{T} \frac{\lambda_{k} F(k)}{a+\|\hat{\varphi}(k)\|^{2}} \hat{\varphi}(k)\left\{y_{0}-y(k)\right\} \\
& =\lambda_{k} F(k) \Delta_{k}\left\{y_{0}-y(k)\right\} .
\end{aligned}
$$

Consequently we have

$$
\begin{aligned}
y_{0}-y(k+1) & =y_{0}-y(k)-\lambda_{k} F(k) \Delta_{k}\left\{y_{0}-y(k)\right\} \\
& =\left(1-\lambda_{k} F(k) \Delta_{k}\right)\left\{y_{0}-y(k)\right\} .
\end{aligned}
$$

Generally, for the positive integer $h$, we have

$$
\left|y_{0}-y(k+h)\right|=\prod_{j=0}^{h-1}\left(1-\lambda_{k+j} F(k) \Delta_{k+j}\right)\left|y_{0}-y(k)\right| .
$$

So as long as $\lambda_{k+j} F(k) \geq 0$, we can obtain

$$
\begin{aligned}
& \left|y_{0}-y(k+h)\right| \\
& \quad \leq\left|y_{0}-y(k)\right| \prod_{j=0}^{h-1}\left|1-\lambda_{k+j} F(k) \frac{\|\left.\hat{\varphi}(k)\right|^{2}-\alpha / 2}{\|\left.\hat{\varphi}(k)\right|^{2}+a}\right| \\
& \leq\left|y_{0}-y(k)\right| \prod_{j=0}^{h-1}\left|1-\lambda_{k+j} F(k) \frac{\alpha-\alpha / 2}{\beta+a}\right| \\
& =\left|y_{0}-y(k)\right| \prod_{j=0}^{h-1}\left|1-\lambda_{k+j} F(k) \frac{\alpha}{2(\beta+a)}\right| \\
& \leq\left|y_{0}-y(k)\right| \prod_{j=0}^{h-1}\left|1-\lambda_{k+j} \frac{\alpha \rho}{2(\beta+a)}\right| .
\end{aligned}
$$

It can be seen that, as long as $\lambda_{k+j}=\lambda$ satisfying $\lambda=(\beta+$ a) $/ \alpha \rho$, we have

$$
\begin{aligned}
\left|y_{0}-y(k+h)\right| & \leq\left|y_{0}-y(k)\right|\left(1-\frac{1}{2}\right)^{h} \\
& =\left|y_{0}-y(k)\right|\left(\frac{1}{2}\right)^{h} \longrightarrow 0 \quad(h \longrightarrow \infty) ;
\end{aligned}
$$

that is,

$$
\lim _{h \rightarrow \infty} y(k+h)=y_{0}
$$

The proof is completed.

Theorem 3. Consider the control law:

$$
\begin{aligned}
u(k)= & u(k-1)+\frac{\lambda_{k}}{a+\|\hat{\varphi}(k)\|^{2}} \hat{\varphi}(k) \\
& \times\left\{A+D\left(Y_{k-1}^{k-n}, U_{k-1}^{k-m}, \theta, k\right)\right\}\left(y_{0}-y(k)\right) .
\end{aligned}
$$

Suppose that there exist the constants $\delta>0, \beta>0$ and $N>0$, such that, when $k>N, \varphi(k)$ and its estimation value $\hat{\varphi}(k)$ constantly satisfy

$$
\begin{aligned}
& \delta \leq \varphi(k) \leq \beta, \\
& \delta \leq \hat{\varphi}(k) \leq \beta .
\end{aligned}
$$

Then there exists the constant $\lambda_{k}$ in the control law (36), such that for $A>0$, when the configuration parameter $\theta$ is useable, the output of the system under the effect of the control law constantly holds:

$$
\lim _{h \rightarrow \infty} y(k+h)=y_{0}
$$

Proof. Let

$$
F(k)=A+D\left(Y_{k-1}^{k-n}, U_{k-1}^{k-m}, \theta, k\right) .
$$

Notice that

$$
\Delta_{k}=\frac{\varphi(k)^{T} \hat{\varphi}(k)}{a+\|\hat{\varphi}(k)\|^{2}}
$$

So we have

$$
\frac{\delta^{2}}{a+\beta^{2}} \leq \Delta_{k} \leq \frac{\beta^{2}}{a+\delta^{2}}
$$

From the control law (36) and the universal model (17), we can easily obtain

$$
y_{0}-y(k+h)=\prod_{j=0}^{h-1}\left(1-\lambda_{k+j} F(k) \Delta_{k+j}\right)\left(y_{0}-y(k)\right) .
$$

Therefore

$$
\left|y_{0}-y(k+h)\right|=\prod_{j=0}^{h-1}\left|1-\lambda_{k+j} F(k) \Delta_{k+j}\right|\left|y_{0}-y(k)\right| .
$$

As long as $\lambda_{k+j}>0$, we can derive from the above formula that

$$
\left|y_{0}-y(k+h)\right| \leq\left|y_{0}-y(k)\right| \prod_{j=0}^{h-1}\left|1-\lambda_{k+j} F(k) \frac{\delta^{2}}{a+\beta^{2}}\right| .
$$

Recall the inequality (25); that is,

$$
A+B>F(k)>\rho>0 ;
$$

then we have

$$
\lambda_{k+j} \rho<\lambda_{k+j} F(k)<\lambda_{k+j}(A+B) .
$$

Notice the formula (41), we can further have

$$
\lambda_{k+j} \rho \frac{\delta^{2}}{a+\beta^{2}} \leq \lambda_{k+j} F(k) \Delta_{k} \leq \lambda_{k+j}(A+B) \frac{\beta^{2}}{a+\delta^{2}} .
$$


Thus it can be seen that, as long as we choose $\lambda_{k}=\lambda$ as

$$
\lambda=\frac{a+\beta^{2}}{3 \rho \delta^{2}},
$$

we can obtain

$$
\begin{aligned}
\left|y_{0}-y(k+h)\right|= & \prod_{j=0}^{h-1}\left(1-\lambda_{k+j} F(k) \Delta_{k+j}\right)\left|y_{0}-y(k)\right| \\
& <\prod_{j=0}^{h-1}\left(1-\frac{1}{3}\right)\left|y_{0}-y(k)\right|=\left(\frac{2}{3}\right)^{h} \\
& \times\left|y_{0}-y(k)\right| .
\end{aligned}
$$

So we have

$$
\lim _{h \rightarrow \infty}\left|y_{0}-y(k+h)\right|=0
$$

that is,

$$
\lim _{h \rightarrow \infty} y(k+h)=y_{0}
$$

The proof is completed.

\section{Analysis of Convergent Conditions and Problem Discussion}

Here we will analyze the significance of the conditions in the convergence theorem and discuss the general application form of model-free control law. Two results are given on the convergence, and it can be seen that these results are only the sufficient conditions. If we suppose that the input is one dimension as well as output, these conditions can be concluded into the following.

(1) There exists $N>0$, such that when $k>N, \varphi(k)$ and its estimation value $\hat{\varphi}(k)$ permanently hold:

$$
\begin{aligned}
& \varphi(k)-\hat{\varphi}(k)=\varepsilon(k), \\
& \frac{a}{2} \leq \varepsilon(k) \hat{\varphi}(k) \leq \frac{a}{2}, \\
& a \leq\|\hat{\varphi}(k)\|^{2} \leq b .
\end{aligned}
$$

(2) There exist constants $\delta>0, \beta>0$ and $N>0$, such that, when $k>N, \varphi(k)$ and its estimation value $\hat{\varphi}(k)$ permanently hold:

$$
\begin{aligned}
& \delta \leq \varphi(k) \leq \beta, \\
& \delta \leq \hat{\varphi}(k) \leq \beta .
\end{aligned}
$$

Each of conditions (1) and (2) is sufficient to make the control law (36) converge.

According to [4], $\varphi(k)$ is the approximate of $(\partial y(k+$ $1)) /(\partial u(k))$ in a sense, and $\hat{\varphi}(k)$ is the estimation value of $\varphi(k)$. In the engineering, $\varphi(k)$ is actually the grads (derivation) of the output $y(k+1)$ to the input $u(k)$ of the system, so the engineering practical significance of conditions (1) and (2) is obvious.

About condition (1) that the function $D\left[Y_{k-1}^{k-n}, U_{k-1}^{k-m}, \theta, k\right]$ satisfies, because $\theta=0$ means that the function $D\left[Y_{k-1}^{k-n}\right.$, $\left.U_{k-1}^{k-m}, \theta, k\right]$ does not contain any element function, we have

$$
D\left[Y_{k-1}^{k-n}, U_{k-1}^{k-m}, \theta, k\right]=0 .
$$

About condition (2), because $D\left[Y_{k-1}^{k-n}, U_{k-1}^{k-m}, \theta, k\right]$ is composed of the mathematical expressions of element functions by their algebraic operation, it is a continuous function of $\theta$, and the continuity is consistent to the remaining variable. This requirement is natural.

We will preliminarily discuss the other form easy to apply of model-free control law. The expression is

$$
\begin{aligned}
u(k)= & u(k-1)+\frac{\lambda_{k}}{a+\|\hat{\varphi}(k)\|^{2}} \hat{\varphi}(k) \\
& \times\left\{A\left(y_{0}-y(k)\right)+G\left(Y_{k-1}^{k-n}, U_{k-1}^{k-m}, \theta, k\right)\right\} .
\end{aligned}
$$

Considering the form easy to analyze, the expression is given by

$$
\begin{aligned}
u(k)= & u(k-1)+\frac{\lambda_{k}}{a+\|\hat{\varphi}(k)\|^{2}} \hat{\varphi}(k) \\
& \times\left\{A+D\left(Y_{k-1}^{k-n}, U_{k-1}^{k-m}, \theta, k\right)\right\}\left(y_{0}-y(k)\right) .
\end{aligned}
$$

It can be seen that as long as we let

$$
D\left[Y_{k-1}^{k-n}, U_{k-1}^{k-m}, \theta, k\right]\left(y_{0}-y(k)\right)=G\left[Y_{k-1}^{k-n}, U_{k-1}^{k-m}, \theta, k\right]
$$

the control law (56) can be changed into (55), but $G\left[Y_{k-1}^{k-n}, U_{k-1}^{k-m}, \theta, k\right]$ need not to be expressed by the form:

$$
D\left[Y_{k-1}^{k-n}, U_{k-1}^{k-m}, \theta, k\right]\left(y_{0}-y(k)\right) .
$$

So form (55) has more universality than form (56). Of course the convergence of form (55) should be analyzed, but it is not carried out in this paper.

\section{Conclusion}

Model-free control law is designed by using the traditional PID controller for reference, which does not depend on the mathematical model of controlled object. Through the new understanding of the P, I, D control functions, we proposed the functional combination idea for controller's design. We can obtain the general form of model-free control law by adding the functional combination part on the basic form. The improved model-free control law is a nonlinear controller, which breaks through the linear combination framework of PID. It is widely used in the actual production process control. Some complex systems that are not well 
controlled by PID can basically realize stability control by using general form of model-free control law.

In this paper, we have introduced the general form of model-free control law and analyzed the convergence of one form. It is shown that the conditions of the convergence theorem can be satisfied in the engineering. There are some other researches on convergence of model-free control law $[23,24]$, but in general, the results of convergence we have obtained are still preliminary. This shows that the theory research of model-free control law is behind its applied research currently.

\section{Acknowledgments}

This work is supported by the Key Laboratory of Electronics Engineering, College of Heilongjiang Province Foundation under Grant DZZD20100023, and by the Student Innovation Laboratory Foundation of Heilongjiang University under Grant CX11143.

\section{References}

[1] Z. G. Han, "The designing problem of model free controller," Control Engineering of China, vol. 9, no. 3, pp. 19-22, 2002.

[2] Z. G. Han, "Study on non-modelling control method for a class of complex systems," Control and Decision, vol. 18, no. 4, pp. 398-402, 2003.

[3] A. P. Jiang, X. Y. Li, and Z. G. Han, "From PID to model-free controller," Control Engineering of China, vol. 12, no. 3, pp. 217-220, 2005.

[4] Z. G. Han, "Integrated approach to modeling and adaptive control," Zidonghua Xuebao/Acta Automatica Sinica, vol. 30, no. 3, pp. 380-389, 2004.

[5] A. P. Jiang, Z. G. Han, and D. Han, "Application of model free control systems to temperature control of heat furnace," Control Engineering of China, vol. 11, no. 5, pp. 388-391, 2004.

[6] Z. G. Han, X. Y. Li, and W. G. Liao, "Application of model free control system to fouth-octanlo device," Control Engineering of China, vol. 16, no. 5, pp. 535-542, 2009.

[7] Z. G. Han, "Model free control law in the production of chemical fertilizer," Control Theory and Applications, vol. 21, no. 6, pp. 858-863, 2004.

[8] Z. G. Han, A. P. Jiang, and G. Q. Wang, "Control system of $\mathrm{H}-\mathrm{N}$ ratio in the production of synthetic ammonia," Control Theory and Applications, vol. 22, no. 5, pp. 762-766, 2005.

[9] P. Ma, W. Li, G. W. Zheng, S. Y. Zhang, and W. W. Ning, "Main steam temperature control system based on MFAC," Electric Power Science and Engineering, no. 1, pp. 19-21, 2006.

[10] X. X. Jiang, H. Wu, G. Zeng, and Z. G. Han, "Application study of model free control method," Control Engineering of China, vol. 14, no. 1, pp. 24-26, 2007.

[11] Z. G. Han, "The application of model free controller," Control Engineering of China, vol. 9, no. 4, pp. 22-25, 2004.

[12] Z. G. Han, "The progress of the theory and application of model free controller," Techniques and Application of Automation, vol. 23, no. 2, pp. 13-16, 2004.

[13] Z. G. Han, "The approach of functional combination of the controller design for large complex system," Control Engineering of China, vol. 11, no. 2, pp. 103-107, 2004.

[14] Z. G. Han and G. Q. Wang, "Cascade scheme of model free control law and its application," Acta Automatica Sinica, vol. 32, no. 3, pp. 345-352, 2006.

[15] A. P. Jiang, X. Y. Li, and H. Wu, "Control function analysis of model free control law," Control Engineering of China, vol. 14, no. 1, pp. 14-17, 2007.

[16] X. Y. Li, Y. Shen, and Z. G. Han, "Optimal design of controller based on functional combination," in Proceedings of the 7th Asian Control Conference (ASCC '09), pp. 1375-1380, Hong Kong, August 2009.

[17] J. Y. Xue, L. Tu, and Z. G. Han, "Performance analysis of model free control method," Control Engineering of China, vol. 16, no. 5, pp. 531-542, 2009.

[18] Q. B. Luo, X. Y. Li, and Z. G. Han, "Analysis of disturbanceresistant ability of model free control method," Journal of System Simulation, vol. 20, no. 13, pp. 3472-3476, 2008.

[19] J. Y. Xue, H. Wu, and Z. G. Han, "On study of model free control system applied to complex large scale time delay systems," Techniques and Application of Automation, vol. 23, no. 4, pp. 1-6, 2004.

[20] Z. G. Han, A. P. Jiang, and G. Q. Wang, "Study on control of multivariable coupling systems with model free control method," Control and Decision, vol. 19, no. 10, pp. 1155-1162, 2004.

[21] A. P. Jiang, X. Y. Li, and Z. G. Han, "Constituting and analyzing the functional combination of model free control law," Control Engineering of China, vol. 13, no. 5, pp. 494-497, 2006.

[22] Z. G. Han, A. P. Jiang, and H. Q. Wang, Adaptive Identification, Prediction and Control-the Multi-Level Recursive Approach, Heilongjiang Education Press, Heilongjiang, China, 1995.

[23] Z. G. Yu and J. Zhao, "Convergence analysis of model free control law with primary pattern," Control Engineering of China, vol. 16, no. 2, pp. 130-132, 2009.

[24] T. Z. Zhang and Z. G. Han, "The convergence analysis of the general form of model free controller," Electric Machines and Control, vol. 10, no. 3, pp. 333-340, 2006. 

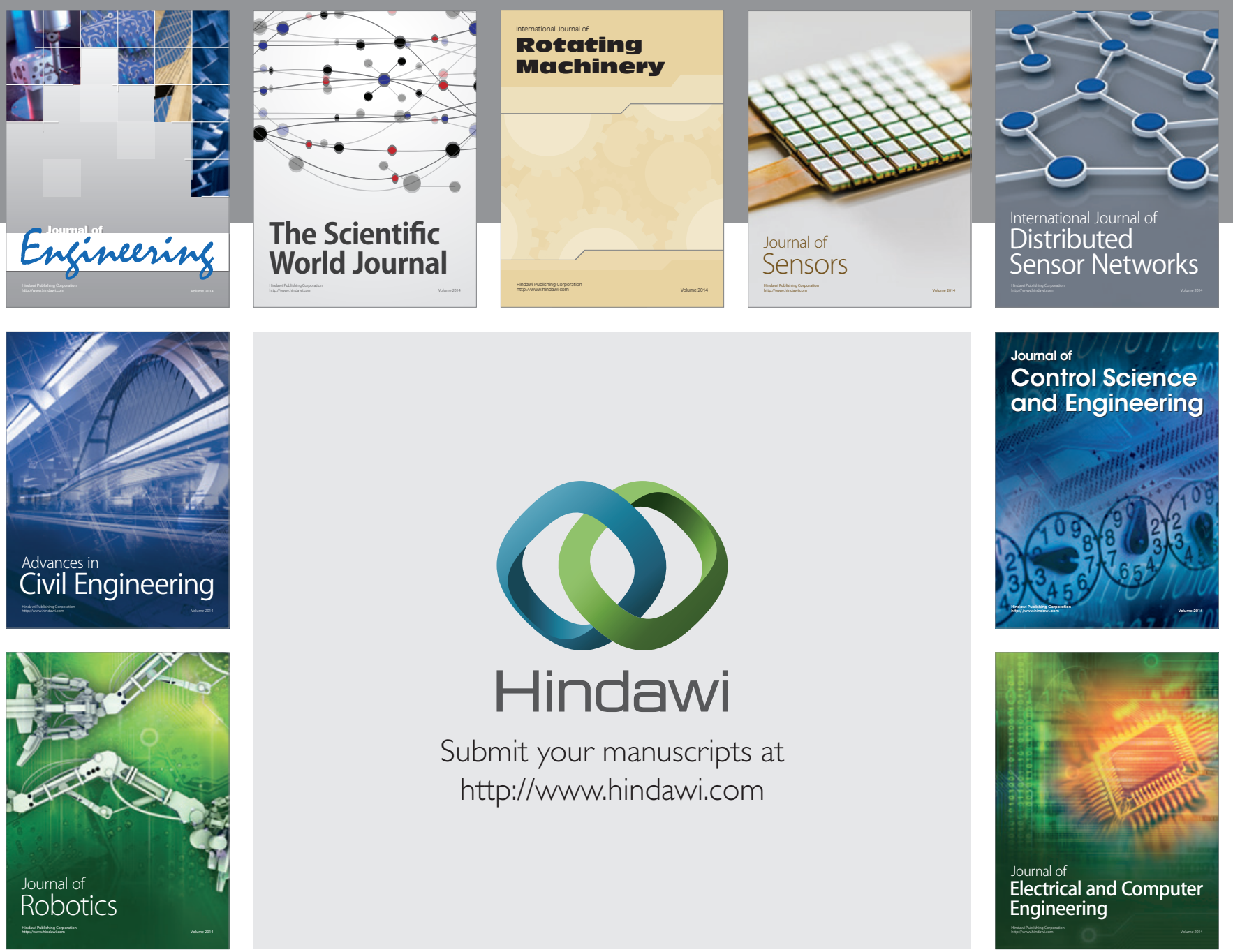

Submit your manuscripts at

http://www.hindawi.com
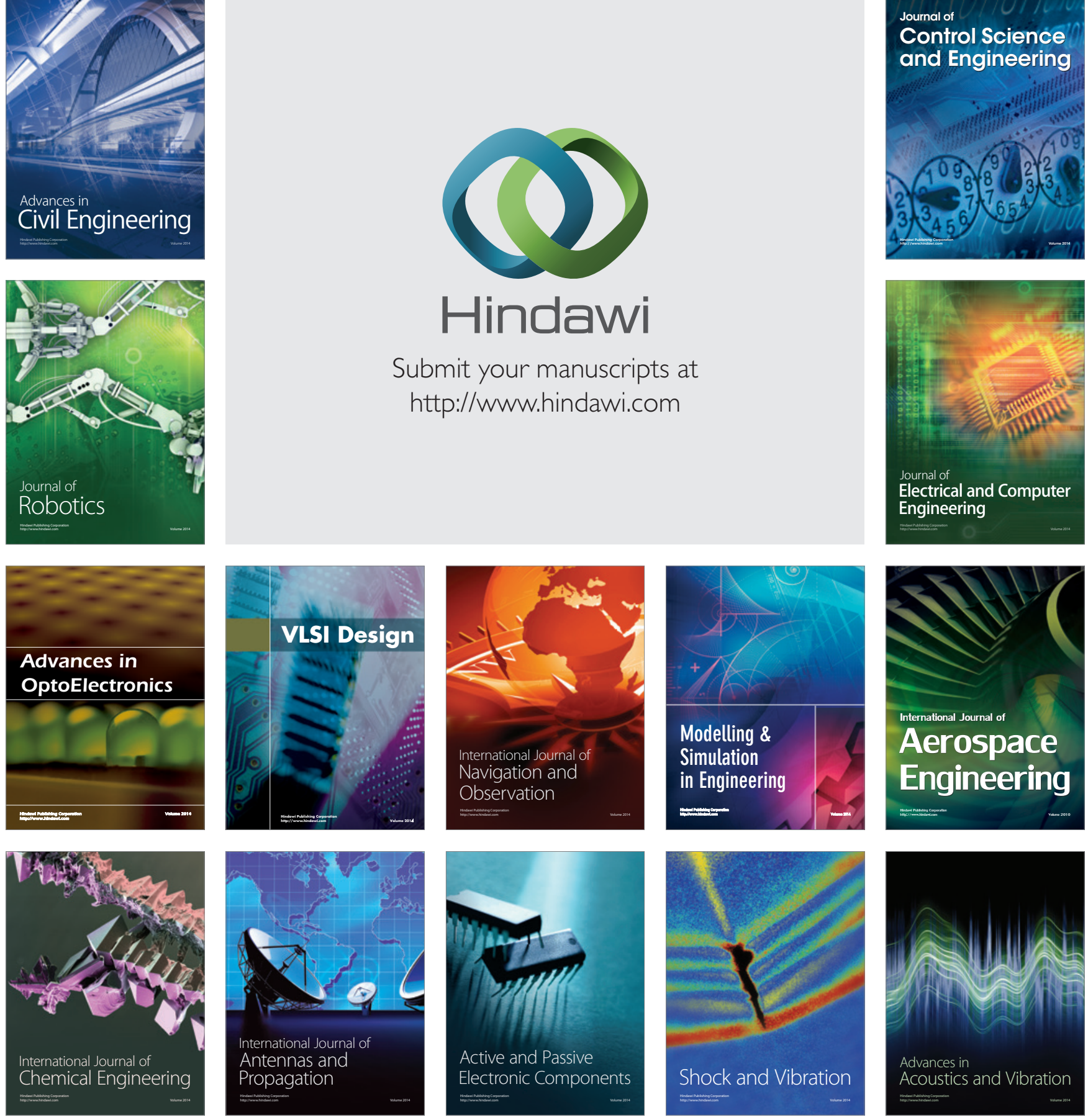